\title{
Structural Insights into Aberrant Topological Patterns of Large-Scale Cortical Networks in Alzheimer's Disease
}

\author{
Yong He, Zhang Chen, and Alan Evans \\ McConnell Brain Imaging Centre, Montreal Neurological Institute, McGill University, Montreal, Quebec, Canada H3A 2B4
}

\begin{abstract}
Recent research on Alzheimer's disease $(\mathrm{AD})$ has shown that cognitive and memory decline in this disease is accompanied by disrupted changes in the coordination of large-scale brain functional networks. However, alterations in coordinated patterns of structural brain networks in $\mathrm{AD}$ are still poorly understood. Here, we used cortical thickness measurement from magnetic resonance imaging to investigate large-scale structural brain networks in $92 \mathrm{AD}$ patients and 97 normal controls. Brain networks were constructed by thresholding cortical thickness correlation matrices of 54 regions and analyzed using graph theoretical approaches. Compared with controls, AD patients showed decreased cortical thickness intercorrelations between the bilateral parietal regions and increased intercorrelations in several selective regions involving the lateral temporal and parietal cortex as well as the cingulate and medial frontal cortex regions. Specially, AD patients showed abnormal small-world architecture in the structural cortical networks (increased clustering and shortest paths linking individual regions), implying a less optimal topological organization in AD. Moreover, AD patients were associated with reduced nodal centrality predominantly in the temporal and parietal heteromodal association cortex regions and increased nodal centrality in the occipital cortex regions. Finally, the brain networks of AD were about equally as robust to random failures as those of controls, but more vulnerable against targeted attacks, presumably because of the effects of pathological topological organization. Our findings suggest that the coordinated patterns of cortical morphology are widely altered in AD patients, thus providing structural evidence for disrupted integrity in large-scale brain networks that underlie cognition. This work has implications for our understanding of how functional deficits in patients are associated with their underlying structural (morphological) basis.
\end{abstract}

Key words: small world; connectivity; cortical thickness; association cortex; betweenness; Alzheimer's disease

\section{Introduction}

Alzheimer's disease (AD) is a progressive, neurodegenerative disease characterized by the decline of cognitive and memory functions. Current theories of AD have posited that the decline arises from alterations in functional integration of distributed brain systems or from structural disconnection between regions because of white matter damage (for review, see Delbeuck et al., 2003). Evidence from previous neurophysiological and neuroimaging studies has shown $\mathrm{AD}$-associated abnormalities not only in the functional connection of several specific brain regions, involving the prefrontal (Horwitz et al., 1987; Grady et al., 2001, 2003), hippocampus (Celone et al., 2006; Wang et al., 2006), cingulate (Greicius et al., 2004), and visual regions (Horwitz et al., 1995; Bokde et al., 2006), but also in the functional integration of the entire brain networks (Stam et al., 2006, 2007). There is also growing evidence to suggest $\mathrm{AD}$-related abnormalities in the structural integrity of white matter such as corpus callosum, su-

Received 0ct. 19, 2007; accepted Feb. 25, 2008.

This work was supported by Human Brain Project Grant P01MH052176-11 (International Consortium for Brain Mapping; principal investigator, Dr. John Mazziotta), Canadian Institutes of Health Research Grant MOP-34996, and the Killam Foundation. Y.H. was supported by a Jeanne Timmins Costello Postdoctoral Fellowship of the Montreal Neurological Institute. We are grateful to Drs. Randy Buckner and Daniel S. Marcus for providing the Open Access Series of Imaging Studies dataset (http://www.oasis-brains.org) for this project.

Correspondence should be addressed to Alan Evans, McConnell Brain Imaging Centre, Montreal Neurological Institute, Montreal, Quebec, Canada H3A 2B4. E-mail: alan.evans@mcgill.ca.

D01:10.1523/JNEUROSCI.0141-08.2008

Copyright $\odot 2008$ Society for Neuroscience $\quad$ 0270-6474/08/284756-11\$15.00/0 perior longitudinal fasciculus, and cingulum (Rose et al., 2000; Medina et al., 2006). In the current study, we focus on a specific issue that remains to be addressed relevant to alterations in the coordinated patterns of large-scale structural brain networks that are constructed using brain morphological features from structural magnetic resonance imaging (MRI).

Recent studies have suggested that there are coordinated variations in brain morphology (e.g., volume and thickness of gray matter) between regions of functionally or anatomically connected systems, such as the frontoparietal (Wright et al., 1999), frontotemporal (Lerch et al., 2006), visual system (Andrews et al., 1997), and symmetrically interhemispheric regions (Mechelli et al., 2005). The notion of morphological coordination has been widely used to study correlated evolution in mammalian brain structures (Barton and Harvey, 2000; Clark et al., 2001) or infer structural connectivity between brain regions in humans (Bullmore et al., 1998; McAlonan et al., 2005; Lerch et al., 2006; Bohbot et al., 2007). The advances in the brain morphological studies have provided a new avenue of research toward large-scale topological organization of the entire human cortex. In our previous study, using graph theoretical network analysis (GRETNA), we demonstrated that coordinated variations in the thickness of the human cerebral cortex at a macroscale (regional level) is neither regular nor random but "small world" in nature (He et al., 2007b).

The small world, characterized by a high degree of clustering 
and short path length linking individual network nodes (Watts and Strogatz, 1998), is an attractive model for the description of complex brain networks because it not only supports both specialized and integrated information processing (Sporns et al., 2004) but also minimizes wiring costs while maximizing the efficiency of information propagation (Kaiser and Hilgetag, 2006; Achard and Bullmore, 2007). Moreover, it provides a quantitative insight into relevant network parameters that have profound effects on the dynamic performances of a network such as the speed of information transfer and robustness against pathological attacks by disease (Bassett and Bullmore, 2006). Recently, using electroencephalogram (EEG) recording, Stam et al. (2007) have reported the loss of small-world characteristics in functional brain networks in $\mathrm{AD}$, implicating disruptive system integrity associated with specific cognitive states caused by the disease. Yet no studies reported $\mathrm{AD}$-related changes in coordinated patterns of large-scale structural brain networks.

Here, we hypothesize that $\mathrm{AD}$ patients would show alterations in the coordination of large-scale structural brain networks, using interregional correlation of cortical thickness as a particular metric of the structural basis underlying brain dynamics. We expected that the abnormalities in the patients with $\mathrm{AD}$ would be associated with alterations in cortical thickness correlations, small-world parameters, nodal centrality, and network robustness because all reflect the precise coordination of cortical morphology in the brain.

\section{Materials and Methods Subjects}

One hundred and ninety-eight right-handed subjects were selected from the Open Access Series of Imaging Studies (OASIS) database (http:// www.oasis-brains.org) (Marcus et al., 2007). Data from nine subjects (one healthy elder adult and eight $\mathrm{AD}$ subjects) were excluded from the analysis because of failure of imaging processing. The included subjects were classified into healthy elders $(n=97)$ and early-stage AD patients ( $n=92$ ) by using the Clinical Dementia Rating (CDR) scale (Morris, 1993; Morris et al., 2001). Sixty-four of the 92 AD patients who had a CDR score of 0.5 were assigned to the very mild category, whereas the other 28 who had a CDR score of 1 were assigned to the mild category. The AD patients (female/male, 54:38) ranged in age from 62 to 96 (mean age, $76.65 ; \mathrm{SD}, 7.13$ ). The healthy controls (female/male, 71:26) ranged in age from 60 to 94 (mean age, 75.93; SD, 9.03). Cognitive function of all subjects was evaluated using the Mini Mental State Examination (MMSE) (Folstein et al., 1975). The AD and control groups had an average MMSE score of 24.38 (range, 14-30) and 28.95 (range, 25-30), respectively. For the details of clinical and demographic data for all subjects, see Marcus et al. (2007).

\section{Image acquisition}

For each subject, three to four individual T1-weighted magnetization prepared rapid gradient-echo (MP-RAGE) images were acquired on a 1.5 T Vision scanner (Siemens, Erlangen, Germany) within a single session. Head movement was minimized by cushioning and a thermoplastic face mask. The images were motion corrected and averaged to create a single image with high contrast-to-noise. The MP-RAGE parameters were empirically optimized for gray-white contrast (repetition time, $9.7 \mathrm{~ms}$; echo time, $4.0 \mathrm{~ms}$; inversion time, $20 \mathrm{~ms}$; delay time, $200 \mathrm{~ms}$; flip angle, $10^{\circ}$; orientation, sagittal; resolution, $256 \times 256$ matrix; slices, 128 ; thickness, $1.25 \mathrm{~mm})$.

\section{Measurements of cortical thickness}

Our methods to automatically measure cortical thickness have been described previously (MacDonald et al., 2000; Kim et al., 2005). Briefly, the native MRI were first registered into stereotaxic space (Talairach and Tournoux, 1988) using a linear transformation (Collins et al., 1994). Simultaneously, the images were corrected for nonuniformity artifacts using the N3 algorithms (Sled et al., 1998). The registered and corrected images were further segmented into gray matter, white matter, CSF, and background using an advanced neural net classifier (Zijdenbos et al., 2002). The inner and outer gray matter surfaces with a total of 81,920 polygons (40,962 vertices) each were then automatically extracted from each magnetic resonance (MR) volume using the constrained Laplacianbased automated segmentation with proximities (CLASP) algorithm (MacDonald et al., 2000; Kim et al., 2005). Cortical thickness was thus defined as the distance between linked vertices on the inner and outer surfaces (Lerch and Evans, 2005). Two representative cortical thickness maps are shown in Figure $1 A$. The CLASP algorithm has been validated using both manual measurements (Kabani et al., 2001) and simulation approaches (Lerch and Evans, 2005; Lee et al., 2006) and applied to recent AD studies (Lerch et al., 2005; Singh et al., 2006).

\section{Construction of structural cortical networks}

We developed a procedure to construct structural cortical networks based on cortical thickness measurements from MRI (He et al., 2007b). First, individual cortical thickness maps were parcellated using the automated nonlinear image matching and anatomical labeling (ANIMAL) package (Collins et al., 1995; Robbins et al., 2004). By registering each subject's MR images to a presegmented volumetric template using nonlinear deformations, the labels of brain regions were transformed to the cortical surface by assigning the value of the voxel label to each vertex on the surface. An average cortical parcellation with 27 regions for each hemisphere was generated by finding the anatomical label with the highest occurrence at each vertex (see Fig. $1 B$; supplemental Table 1, available at www.jneurosci.org as supplemental material). Cortical thickness for each region was measured as the average thickness of all vertices defined as belonging to that region. The interregional correlation matrix $C_{i j}(i$, $j=1,2, \ldots N$, here $N=54$ ) of each group (see Fig. $1 C$, left for the control group and right for the $\mathrm{AD}$ group) was then obtained by calculating the partial correlation coefficients across individuals between the cortical thicknesses of every pair of regions. The partial correlations between any two cortical regions represent their conditional dependences by partialling out the effects of the other 52 regions defined in the ANIMAL template (Horwitz et al., 1987; Salvador et al., 2005). Before the correlation analysis, a linear regression was performed at every cortical region to remove the effects of age and gender; the resulting residuals were used to substitute for the raw cortical thickness values. Finally, the partial correlation matrix of each group was thresholded into a binarized matrix $A_{i j}=$ $\left[a_{i j}\right]$, where $a_{i j}$ was 1 if the absolute value of the correlation $C_{i j}$ between regions $i$ and $j$ was larger than a given correlation threshold and 0 otherwise. These binary matrices capture the underlying topological organization of the human structural cortical networks. Notably, it is also possible to use the continuous weighting (e.g., true correlation values) between regions to study the network properties (Barrat et al., 2004; Jiang et al., 2004), but it would lead to complicated statistical descriptions in the following network analysis. The current study therefore confined itself to a simpler binary graph theoretical analysis.

\section{Graph theoretical approaches}

Threshold selection. The network (graph) $G$ was represented by a binarized matrix $A_{i j}$ with $N$ nodes and $K$ edges, where nodes and edges indicate cortical regions and undirected links corresponding to its nonzero elements, respectively. It was noted that, when the same correlation threshold was applied to the correlation matrices of the $\mathrm{AD}$ and control groups, the resulting graphs would comprise different number of edges because of the discrepancies in the low-level correlations (see Fig. 1C, left vs right). Thus, between-group difference in network parameters would not purely reflect the alterations in the topological organization. To control this effect, we thresholded the correlation matrix $C_{i j}$ of each group into a binarized matrix with a fixed sparsity $S$ (see Fig. $1 D$, left for the control group and right for the $\mathrm{AD}$ group) defined as the total number of edges $K$ in a graph divided by the maximum possible number of edges $N(N-1) / 2$. Setting a sparsity-specific threshold ensures that the graphs of both groups have the same number of edges or wiring cost (Achard and Bullmore, 2007; Stam et al., 2007). However, because there is currently no definitive way to select a single threshold, we therefore thresholded each correlation matrix repeatedly over a wide range of sparisty 
$(6 \% \leq S \leq 40 \%)$ and then estimated the properties of the resulting graphs at each threshold value. This enabled us to compare the smallworld parameters between the two groups as a function of sparsity independently of the precise selection of threshold. The range of sparsity was chosen here to allow small-world network properties to be properly estimated and the number of spurious edges in each network minimized as indicated in previous studies (Achard and Bullmore, 2007; He et al., 2007b).

Small-world analysis. Small-world measures of a network (clustering coefficient, $C_{p}$, and characteristic path length, $L_{p}$ ) were originally proposed by Watts and Strogatz (1998). Briefly, the $C_{p}$ is the average of the clustering coefficients over all nodes in a network, where the clustering coefficient $C_{i}$ of a node $i$ is defined as the number of existing connections among the neighbors of the node divided by all their possible connections. $C_{p}$ quantifies the extent of local cliquishness or local efficiency of information transfer of a network (Watts and Strogatz, 1998; Latora and Marchiori, 2001). The $L_{p}$ of a network is the average minimum number of connections that link any two nodes of the network. However, this original definition of $L_{p}$ is problematic in networks that comprise more than one component because there exist nodal pairs that have no connecting path. To avoid this problem, $L_{p}$ was measured here by using a "harmonic mean" distance between pairs proposed by Newman (2003) (i.e., the reciprocal of the average of the reciprocals). $L_{p}$ quantifies the ability of parallel information propagation or global efficiency (in terms of $1 / L_{p}$ ) of a network (Latora and Marchiori, 2001). A real network would be considered small world if it meets the following criteria: $\gamma=C_{p}^{\text {real }}$, $C_{p}^{\text {rand }}>1$ and $\lambda=L_{p}^{\text {real }} / L_{p}^{\text {rand }} \sim 1$ (Watts and Strogatz, 1998), where the $C_{p}^{\text {rand }}$ and $L_{p}^{\text {rand }}$ are the mean clustering coefficient and characteristic path length of matched random networks that preserve the same number of nodes, edges, and degree (the degree $k_{i}$ of a node $i$ is the number of connections to that node) distribution as the real network (Maslov and Sneppen, 2002; Sporns and Zwi, 2004).

Nodal centrality. In this study, we also investigated nodal characteristics of the cortical networks. To address this issue, we considered the "betweenness centrality" of the nodes in the networks. The betweenness $B_{i}$ of a node $i$ is defined as the number of shortest paths between any two nodes that run through node $i$ (Freeman, 1977). We measured the normalized betweenness as $b_{i}=B_{i} /\langle B\rangle$, where $\langle B\rangle$ was the average betweenness of the network. $b_{i}$ is a global centrality measure that captures the influence of a node over information flow between other nodes in the network and it was calculated here by using the MatlabBGL package (http://www.stanford.edu/ dgleich/programs/matlab_bgl/). The hubs of the cortical networks are the regions with high values of $b_{i}$. Group differences in $b_{i}$ reflect the effects of the disease on the global roles of regions in the cortical networks.

Network robustness. Network robustness, characterized by the degree of tolerance against random failures and targeted attacks, is usually associated with the stability of a complex network. In the present study, we investigated the robustness (tolerance) of the cortical networks by the removals of nodes and edges (Albert et al., 2000; Kaiser and Hilgetag, 2004; Achard et al., 2006). To address the nodal failure tolerance, we first randomly removed one node from the networks and then measured the changes in the size of the largest connected component. After this, we continued selecting and removing additional nodes from the networks at random and recomputed this measure. This procedure was also applied to the edge failure tolerance assessment in which the links in the networks were randomly selected and removed. To evaluate the attack tolerance, we repeated the above processes but removed the nodes and links in decreasing order of their betweenness, respectively [the betweenness of an edge is the number of shortest paths between pairs of other nodes that pass through the edge (Girvan and Newman, 2002); links with high edge betweenness values can be regarded as the pivotal links of a network]. The simulation procedures were performed for the networks of both control and $\mathrm{AD}$ groups, and a comparison of the results was further investigated.

\section{Statistical analysis}

Correlation differences. To test whether interregional correlation of cortical thickness was significantly different between the $\mathrm{AD}$ patients and controls, correlation coefficients obtained above were further converted into $z$ values by using Fisher's $r$-to- $z$ transform. This transformation generated values that were approximately normally distributed. A $Z$ statistic was then used to compare these transformed $z$ values to determine the significance of the between-group differences in correlations (Cohen and Cohen, 1983). To adjust for the multiple comparisons, a false discovery rate (FDR) procedure was performed at a $q$ value of 0.05 (Genovese et al., 2002).

Topological parameters differences. To determine statistical significance of the differences in network parameters between groups, a nonparametric permutation test method was applied (Bullmore et al., 1999). First, $C_{p}$ $L_{p}$, and $b_{i}$ of the networks at a given sparsity were computed separately for the $\mathrm{AD}$ and control groups. To test the null hypothesis that the observed group differences could occur by chance, we then randomly reallocated each subject's set of regional cortical thickness measures to one or the other of the two groups and recomputed the partial correlation matrix for each randomized group. We then obtained corresponding binarized matrix using the same sparsity threshold as in the real brain networks. Next, we calculated the network parameters for each randomized group and obtained their differences between the randomized groups. This randomization procedure was repeated 1000 times and the 95 percentile points of each distribution were used as the critical values for a one-tailed test of the null hypothesis with a probability of type I error of 0.05 . The procedure was repeated at every sparsity threshold value of the brain networks. In the present study, we also assessed between-group differences in the areas under the $C_{p}$ and $L_{p}$ curves.

\section{Results}

\section{Interregional correlations of cortical thickness}

The interregional cortical thickness correlation matrices of the normal controls and $\mathrm{AD}$ groups are shown in Figure 1C. The correlation patterns of both groups were complex but had some common features such as strong interhemispheric intercorrelations between bilaterally homologous regions that were consistent with previous brain morphological studies using cortical thickness (He et al., 2007b) and gray matter density (Mechelli et al., 2005) in normal subjects. Statistical analysis further revealed significant between-group correlation differences $(p<0.05$, FDR-corrected) in various pairs of cortical regions (Table 1). For instance, decreased interhemispheric positive correlations between the bilateral postcentral gyrus (PoCG) and between the bilateral superior parietal lobe (SPL) were observed in the AD patients compared with the controls. In addition, $\mathrm{AD}$ patients were also found to show increased positive correlations in various pairs of cortical regions involved in the lateral parietal, temporal, and frontal cortices [supramarginal gyrus (SMG), inferior temporal gyrus (ITG), superior temporal gyrus (STG), and middle frontal gyrus (MFG)] as well as the medial frontal gyrus (MdFG) and cingulate (CING) regions. Additionally, we also noted several abnormal negative correlations in the $\mathrm{AD}$ patients (Table 1).

\section{Small-world structural cortical networks}

\section{Small-world model}

Previous studies have demonstrated the small-world topology in large-scale structural brain networks in humans (Hagmann et al., 2007; He et al., 2007b) and nonhuman primates (Hilgetag et al., 2000; Sporns and Zwi, 2004). In a small-world network, the shortest path length between any pair of nodes is approximately equivalent to a comparable random network, but the nodes of the network have greater local interconnectivity than a random network (Watts and Strogatz, 1998). In the current study, we also examined the small-world attributes of cortical networks in the normal elder subjects and AD patients. As expected, both networks (Fig. 2, left for the control group and right for the $\mathrm{AD}$ group) demonstrated small-world architectures as they had an almost identical path length $(\lambda \approx 1$, black lines) but were more 
A
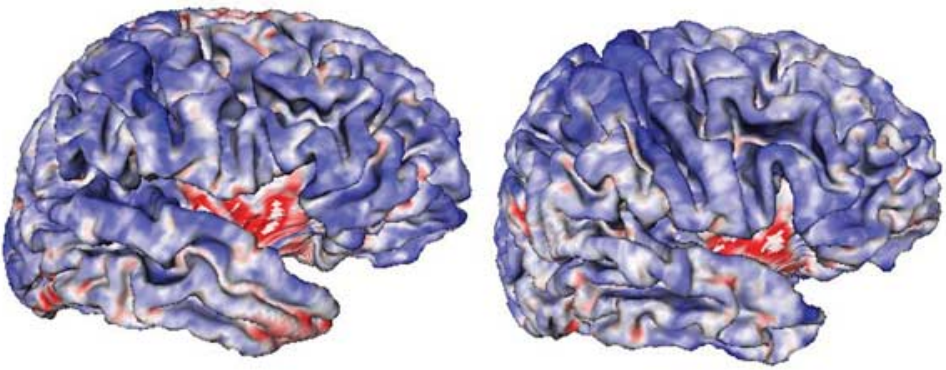

$7 \mathrm{~mm}$
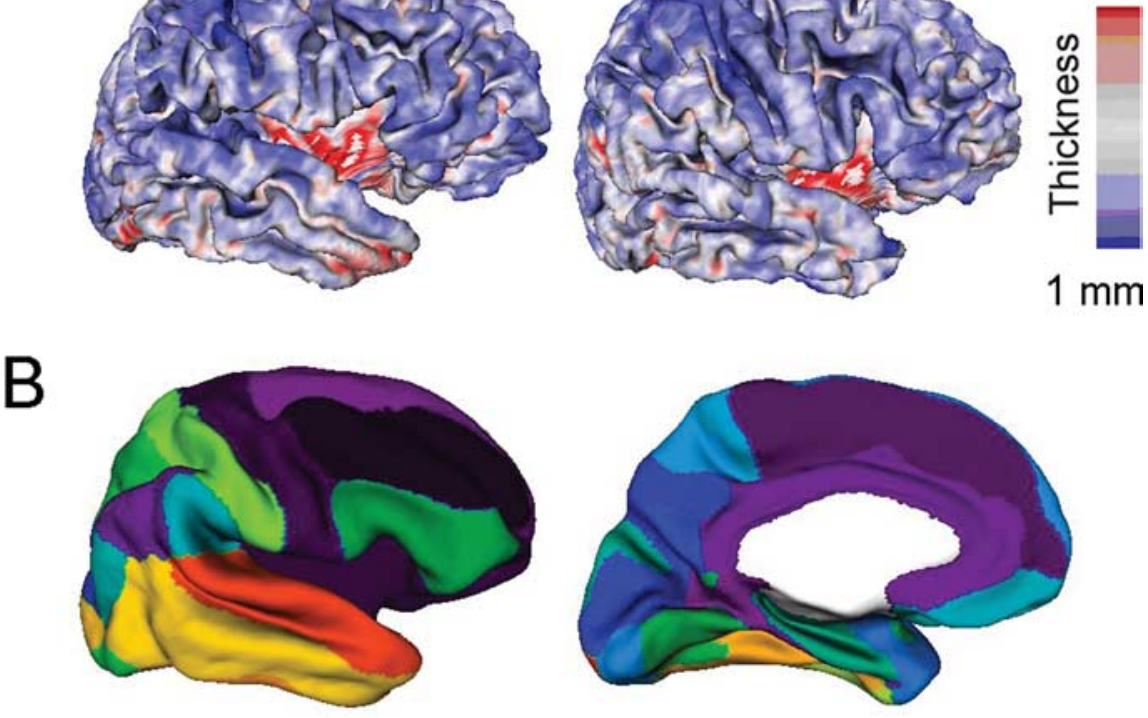

$1 \mathrm{~mm}$

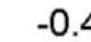
$-0.2$

0

0.2

0.4

0.6

C

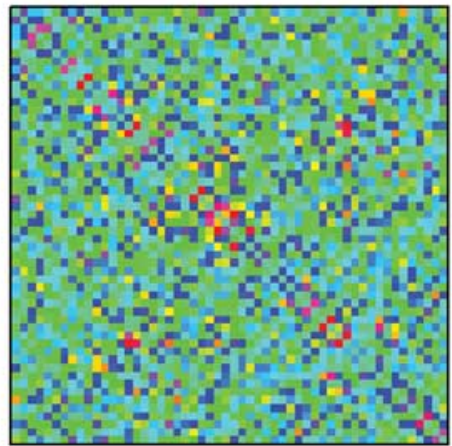

NC

D

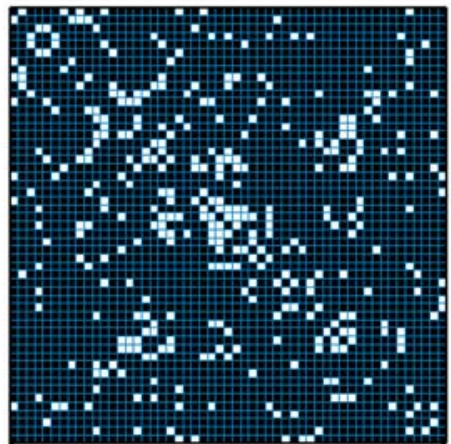

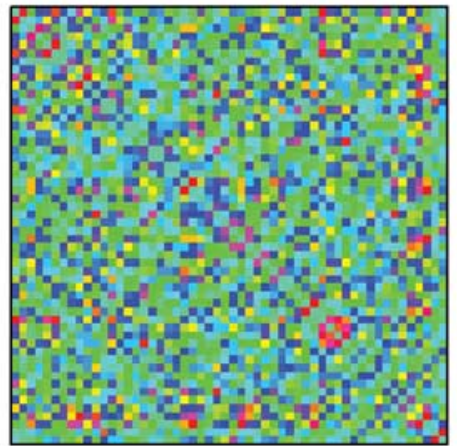

AD

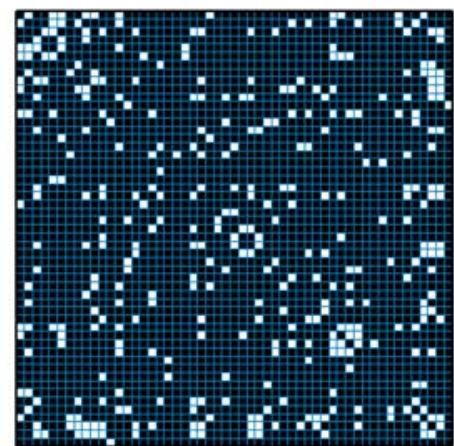

Figure 1. A flowchart for the construction of structural cortical networks. $A$, Two representative cortical thickness maps (left for a control subject and right for an AD subject) were obtained from anatomical MRI by computational neuroanatomy. The color bar indicating the range of thickness is shown on the right. $\boldsymbol{B}$, The entire cerebral cortex was segmented into 54 cortical areas that were displayed on the average cortex (left for the lateral surface and right for the medial surface), each color representing an individual region. $\boldsymbol{C}$, The correlation matrices were obtained by calculating partial correlations between regional thickness across subjects within each group (left for the control group and right for the AD group). The color bar indicating the partial correlation coefficient between regions is shown on the top. $\boldsymbol{D}$, The correlation matrices of $\boldsymbol{C}$ were thresholded into the binarized matrices (left for the control group and right for the AD group) by a sparsity threshold of $13 \%$. Such a threshold ensures that the networks of both of the groups have the same number of nodes and links (i.e., the two networks have the same wiring cost). NC, Normal controls. For details, see Materials and Methods. locally clustered ( $\gamma>1$, gray lines) over a wide range of sparsity $(6 \% \leq S \leq 40 \%)$ in comparison with the matched random networks, consistent with the previous studies. Using computational modeling simulation approaches, Sporns et al. (2000) suggest the emergence of small-world topology when networks are evolved for high complexity of dynamic behavior defined as an optimal balance between local specialization and global integration. Our findings thus provide additional support for the hypothesis that the human brain has evolved into a complex but efficient neural architecture to maximize the power of information processing (Sporns et al., 2004; Kaiser and Hilgetag, 2006).

\section{$A D$-related alterations}

One of the key questions we posed was whether $\mathrm{AD}$ patients with cognitive impairment would be associated with alterations of small-world parameters in the cortical networks. As shown in Figure 3, both clustering coefficient, $C_{p}$ (Fig. $3 A$, inset), and characteristic path length, $L_{p}$ (Fig. $3 B$, inset), in the $\mathrm{AD}$ networks were larger than those of the controls over a wide range of sparsity. Statistical analysis further revealed significant differences $(p<0.05)$ in the $C_{p}$ values at $12 \%<S<15 \%$ and $27 \%$ $<S<40 \%$ (Fig. $3 A$, arrows) and $L_{p}$ values at $12 \%<S<14 \%$ and $22 \%<S<34 \%$ (Fig. 3B, arrows). Moreover, $\mathrm{AD}$ patients demonstrated significantly larger area under the $C_{p}(p=0.03)$ (Fig. $\left.4 A\right)$ and $L_{p}(p=$ 0.05 ) (Fig. $4 B$ ) curves. These results imply that the small-world parameters were significantly altered in the structural cortical networks of $\mathrm{AD}$ patients. Our finding of $\mathrm{AD}$-related increases in paths was consistent with a recent brain functional network study in AD using EEG measurement (Stam et al. 2007). However, the previous study did not detect significant changes in clustering coefficients in $\mathrm{AD}$ patients. The discrepancies could be attributable to different network modality (functional vs structural), network size (21 vs 54), and population size (28 vs 189 ) applied in two studies. Nonetheless, all evidence points to that $\mathrm{AD}$ patients are probably related to the loss of small-world characteristics in the large-scale brain systems.

\section{Regional nodal characteristics}

To investigate the nodal characteristics, the cortical networks were constructed at a specific sparsity threshold of $13 \%$. This threshold ensures that all regions are included in the cortical networks while minimizing the number of false-positive paths (Fig. 5). Such a constraint might optimize 
interregional correlation strengths and therefore be biologically plausible (Bassett et al., 2006).

\section{Hub regions}

To identify the hub regions, we examined normalized nodal betweenness centrality, $b_{i}$, of each cortical region in both networks (see Materials and Methods). In the control group, 11 regions including 8 heteromodal or unimodal association cortex regions, 2 paralimbic cortex regions, and 1 primary motor cortex region were identified as the hubs because of large values in $b$ $\left[b_{i}>1.5\right.$ (i.e., the betweenness value of a node is $>1.5$ times the average betweenness of the network)] (Table 2; supplemental Fig. $1 A$, available at www. jneurosci.org as supplemental material). In the $\mathrm{AD}$ group, 14 regions including 9 heteromodal or unimodal association cortex regions and 5 paralimbic cortex regions were identified as the hubs (Table 3; supplemental Fig. $1 B$, available at www. jneurosci.org as supplemental material). These identified hubs were predominately located in regions of heteromodal and unimodal association cortex [SMG, STG, middle temporal gyrus, middle frontal gyrus, and lingual gyrus (LG)] (Tables 2, 3) receiving convergent inputs from multiple other cortical regions (Mesulam, 1998), suggesting their pivotal roles in the human structural cortical networks. The findings are in accordance with several previous studies in which these recently evolved association cortex regions have been identified as critical nodes in both structural and functional brain networks in humans (Achard et al., 2006; He et al., 2007b) and nonhuman primates (Sporns and Zwi, 2004; Honey et al., 2007).

\section{$A D$-related alterations}

We next examined AD-related changes in the betweenness centrality of cortical regions. Compared with the controls, the $\mathrm{AD}$ patients showed significant centrality decreases in the heteromodal regions of the lateral temporal and parietal cortex [STG and angular gyrus (ANG)] and increases in the unimodal association cortex [LG and lateral occipitotemporal gyrus (LOTG)] and paralimbic regions (CING) (Fig. 6A, Table 4). The involved regions were highlighted in the topographic maps of the networks for both the control (Fig. $6 B$, left) and $\mathrm{AD}$ (Fig. $6 \mathrm{~B}$, right) groups. Additionally, we also noted that the betweenness values of the bilateral cortical regions were highly correlated in the normal subjects (Pearson's $r=0.50, p=0.007$ ) but not in the AD patients (Pearson's $r=0.13, p=0.52$ ). Additional statistical analysis revealed a decreased trend in correlation coefficient of the patients $(Z$ score $=1.47 ; p=0.07$ ) (supplemental Fig. 2 , available at www.jneurosci.org as supplemental material). The result was consistent with previous neuroimaging studies showing increased structural and functional asymmetry in $\mathrm{AD}$ patients (Grady et al., 1986; Haxby et al., 1990). Together, our findings suggest that the roles of regions in managing information flows over the cortical networks were profoundly affected in $\mathrm{AD}$ patients.

\section{Reduced network robustness in $\mathrm{AD}$ patients}

Figure 7 illustrates the network robustness of both the control and $\mathrm{AD}$ groups in response to the random failures and targeted
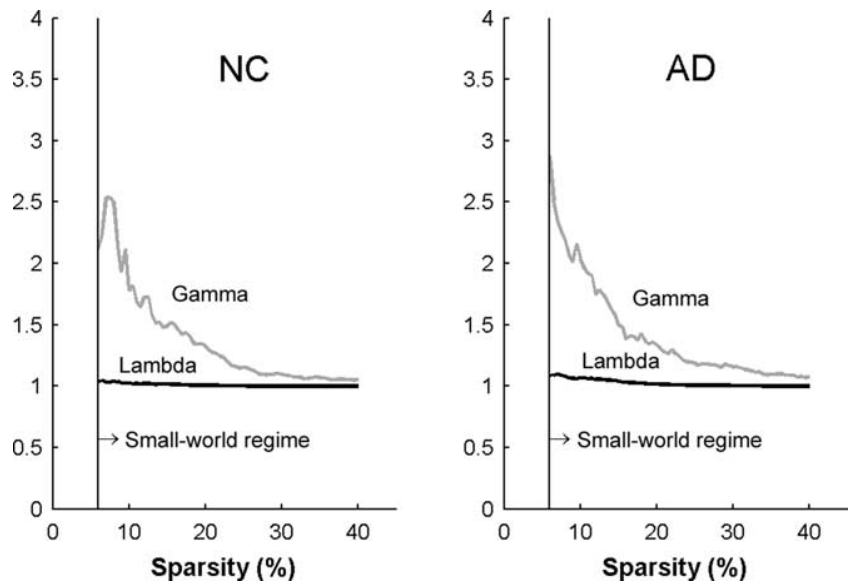

Figure 2. Small-world properties of structural cortical networks. The graphs show the changes in the $\gamma\left(C_{p}^{\text {real }} / C_{p}^{\text {rand }}\right.$, gray lines $)$ and $\lambda\left(L_{p}^{\text {real } /} / L_{p}^{\text {rand }}\right.$, black lines $)$ in the structural networks of both the control (left panel) and AD (right panel) groups as a function of sparisty thresholds. At a wide range of sparsity, both networks have $\gamma>1$ (i.e., the real networks show high clustering compared with 1000 rewiring random networks) and $\lambda \sim 1$ (i.e., the real networks show approximately equivalent path length compared with 1000 rewiring random networks), which implies prominent small-world properties (see Materials and Methods). Note that, as the values of sparsity thresholds increase, the $\gamma$ values decrease rapidly, but the $\lambda$ values only change slightly. The black arrows point to a range of sparsity in which the small-world properties are estimable because the average degrees of networks are larger than $\log (N)(N$ is the number of node regions) (Watts and Strogatz 1998; Achard et al., 2006; He et al., 2007b). NC, Normal controls.

attack. Although the structural cortical network of AD patients was approximately as robust to the random failures as that of controls, it was considerably more vulnerable to targeted attacks. We found that the size of the largest connected component was reduced more noticeably in the network of $\mathrm{AD}$ patients than in the controls when at least $10 \%$ of the most central nodes and links were attacked (Fig. 7, right). Furthermore, when $25 \%$ of the most central nodes were attacked in both networks, the size of the largest component reduced $40 \%$ in the network of $\mathrm{AD}$ patients (Fig. 7A, right arrow); whereas in contrast, the network of controls only reduced 25\% (Fig. 7A, left arrow). Similarly, whereas $25 \%$ of the most central links were attacked, the size of the largest 

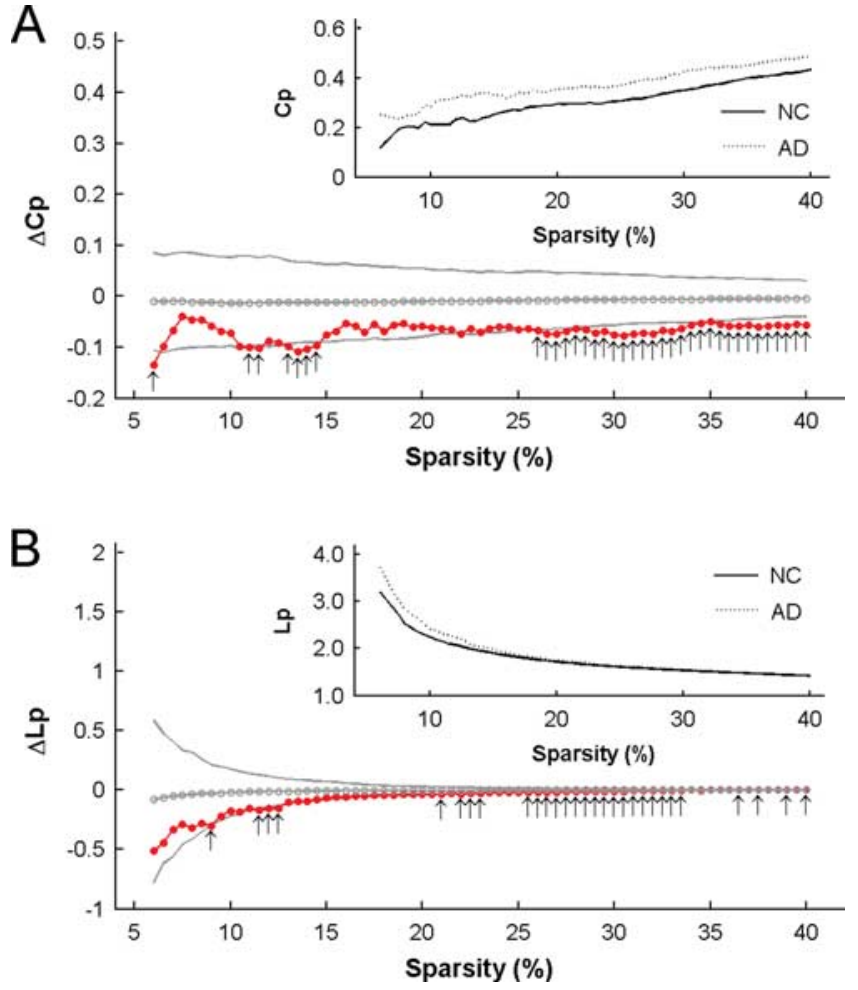

Figure 3. Between-group differences in clustering coefficient $\left(C_{p}\right)$ and path length $\left(L_{p}\right)$ as a function of sparsity. $A$, The graph shows the differences (red circles) in the $C_{p}$ between the controls and AD patients as a function of sparsity thresholds. The gray lines represent the mean values (open circles) and $95 \%$ confidence intervals of the between-group differences obtained 1000 permutation tests at each sparsity value. The arrows indicate significant $(p<0.05)$ difference in $C_{p}$ between the two groups. Note that AD patients (dotted lines) show larger $C_{p}$ values in the brain networks than controls (solid lines) over a wide range of thresholds (inset). $\boldsymbol{B}$ The graph shows the differences (red circles) in the $L_{p}$ between the controls and AD patients as a function of sparsity thresholds. The gray lines represent the mean values (open circles) and $95 \%$ confidence intervals of the between-group differences obtained 1000 permutation tests at each sparsity value. The arrows indicate significant $(p<0.05)$ difference in $L_{p}$ between the two groups. Note that $A D$ patients (dotted lines) show larger $L_{p}$ values in the brain networks than controls (solid lines) over a wide range of thresholds (inset). NC, Normal controls.
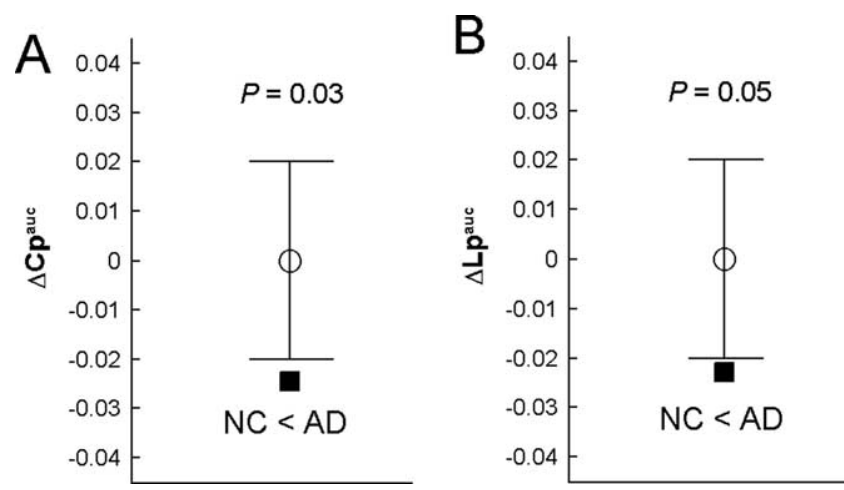

Figure 4. Between-group differences in areas under the clustering coefficient $\left(C_{p}^{\text {AUC }}\right)$ and path length $\left(\mathrm{L}_{p}^{\mathrm{AUC}}\right)$ curves. $A$, The graph shows the differences (black square) in the $C_{p}^{\text {AUC }}$ between the controls and AD patients. The black lines represent the mean values (open circles) and $95 \%$ confidence intervals of the between-group differences obtained 1000 permutation tests. Note that the AD patients show larger $C_{p}^{\text {AUC }}$ values in the brain networks compared with the controls $(p=0.03)$. B , The graph shows the differences (black square) in the $L_{p}^{\text {AUC }}$ between the controls and AD patients. The black lines represent the mean values (open circles) and $95 \%$ confidence intervals of the between-group differences obtained 1000 permutation tests. Note that the AD patients show larger $L_{p}^{\text {AUC }}$ values in the brain networks compared with the controls $(p=0.05)$. For the $C_{p}$ and $L_{p}$ curves of the controls and AD groups, see the insets of Figure 3. NC, Normal controls.

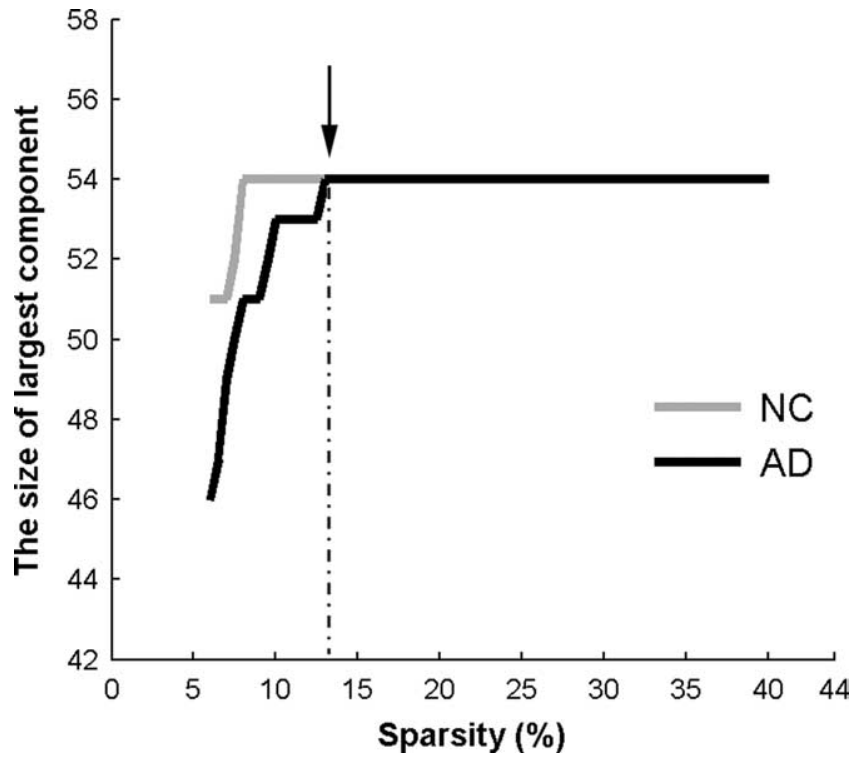

Figure 5. The size of the largest connected component of structural cortical networks. The graph shows the largest component size of the networks in the control (gray line) and AD group (black line) as a function of sparsity threshold. As the threshold increases, the largest component sizes of both groups tend to increase. The arrow indicates that the lowest sparsity threshold (13\%) in which both of the networks included all connected nodes (i.e., 54 regions) defined in the brain template.

component reduced $20 \%$ in the network of $A D$ patients (Fig. $7 B$, right arrow) but it remained nearly unchanged in the control group (Fig. 7B, left arrow). These results suggest that the structural cortical networks of AD patients exhibited topological vulnerability to targeted attack as a consequence of pathological organization.

\section{Discussion}

The present study, for the first time, demonstrates AD-related changes in the coordination of large-scale structural brain networks by using cortical thickness data from MRI. Our main findings are as follows: (1) that the observed data revealed AD-related abnormalities in morphological correlations among selective subsets of cortical regions, (2) that the global topological organization of cortical networks in AD patients were disrupted as indicated by altered small-world parameters, (3) that the regional characteristic (centrality) was profoundly affected in AD patients, and (4) that the structural cortical network of AD showed reduced topological stability while against targeted attack. Together, these findings strongly suggest that the widely distributed cortical networks are altered in $\mathrm{AD}$ patients, thus providing structural (morphological) evidence to support the concept of $\mathrm{AD}$ that the core aspects of the pathophysiology of this disease are associated with disruptive alterations in the coordination of large-scale brain networks that underlie high-level cognition.

\section{Neurobiological basis of correlative variation in regional cortical thickness}

The thickness of the cerebral cortex represents the size, density, and arrangement of cells (Parent and Carpenter, 1995). In this study, interregional correlations in cortical thickness were used to construct structural brain networks. Previous work from our laboratory has demonstrated that interregional coordinated variations in cortical thickness are approximately associated with the known neuroanatomical pathways in the human brain (Lerch et al., 2006; He et al., 2007b). Other morphological covariations 
(e.g., gray matter volume) were also reported in regions of functionally or anatomically connected systems (Andrews et al., 1997; Mechelli et al., 2005; Bohbot et al., 2007). Although the precise neurobiological mechanism behind them remains unclear, it was argued (Mechelli et al., 2005) that the morphological coordination in circumscribed regions may result from the mutually trophic effects (Ferrer et al., 1995) or environment-related plasticity (Maguire et al., 2000). Recent studies have also demonstrated disrupted coordination of the brain morphology in various neuropsychiatric disorders, such as schizophrenia (Bullmore et al., 1998; Wright et al., 1999; Mitelman et al., 2005), autism (McAlonan et al., 2005), and obsessive-compulsive disorder (Pujol et al., 2004). All these processes are the likely driving forces behind alterations in correlative patterns of cortical thickness in AD.

\section{Abnormal interregional correlations of cortical thickness in $\mathrm{AD}$}

We observed several abnormal interregional cortical thickness correlations in AD patients (Table 1). Decreased positive correlations were found between the bilateral parietal regions (PoCG and SPL). Studies in monkeys (Seltzer and Pandya, 1983) and humans (Huang et al., 2005) have demonstrated that the bilateral parietal regions are connected through the midbody (PoCG) and posterior body and splenium (SPL) of the corpus callosum (CC). Several electrophysiological and neuroimaging studies found that the $\mathrm{AD}$ patients had (1) impaired functional couplings between bilateral homologous regions (Horwitz et al., 1987; Wada et al., 1998), and (2) reduced size or integration of white matter in the midbody, posterior body, and splenium of the CC (Pantel et al., 1999; Sydykova et al., 2007). Thus, our findings are compatible with the previous studies of $\mathrm{AD}$. The decreases could be attributed to lack of mutually trophic influences of anatomical connectivity (CC) between the regions.

Interestingly, we found that $\mathrm{AD}$ patient exhibited increased positive correlations among several "default" (Raichle et al., 2001) regions [e.g., medial frontal and parietal (MPFC and CING), and lateral parietal and temporal (SMG, ITG, and STG) regions]. It has been suggested that these regions may be directly connected. Several recent resting-state functional MRI (fMRI) studies have shown that these regions are temporally coherent in intrinsic or spontaneous brain activity in both humans (Greicius et al., 2003; Fox et al., 2005) and monkeys (Vincent et al., 2007). Moreover, these "default" regions have also been found to be mainly involved in episodic memory process (Greicius et al., 2003) and show AD-related breakdown of brain activities, such as the amyloid deposition, metabolic and spontaneous activity disruption (Greicius et al., 2004; Buckner et al., 2005; Celone et al., 2006). In addition, these regions were also found to show focal thinning in $\mathrm{AD}$ patients (supplemental Fig. 3 and supplemental Table 4, available at www.jneurosci.org as supplemental material). One could thus speculate that increased correlations of cor- tical thickness among the "default" regions may reflect a correlative cortical shrinking because of shared vulnerability to an insult, as preferentially affected by the pathological process of this disease.

We also noted abnormal negative correlations in the AD patients. Although the phenomenon of negative morphological correlations has been observed in normal subjects (Mechelli et al., 2005; Bohbot et al., 2007), and patients with schizophrenia (Mitelman et al., 2005) and autism (McAlonan et al., 2005), it remains unclear as to what they precisely reflect. One hypothesis is that the abnormalities are related to weakened or strengthened interregional inhibitory trophic relationship because of a direct influence between the regions or an indirect modulation from a third party, leading to a reorganization of brain systems (McAlonan et al., 2005; Mitelman et al., 2005).

\section{Altered small-world configurations in structural cortical networks in AD}

We also found significant small-world alterations (i.e., increased path length and clustering coefficient in the structural networks of $\mathrm{AD}$ patients). Short paths in brain networks assure effective integrity or rapid transfers of information between and across remotely regions that are believed to constitute the basis of cognitive processes (Sporns and Zwi, 2004). The AD-related increases in paths might thus reflect disrupted neuronal integrations among distant regions that were associated with impaired cognitive functions (measured with MMSE scores). In this study, $\mathrm{AD}$ patients were also found to show increased clustering in the 

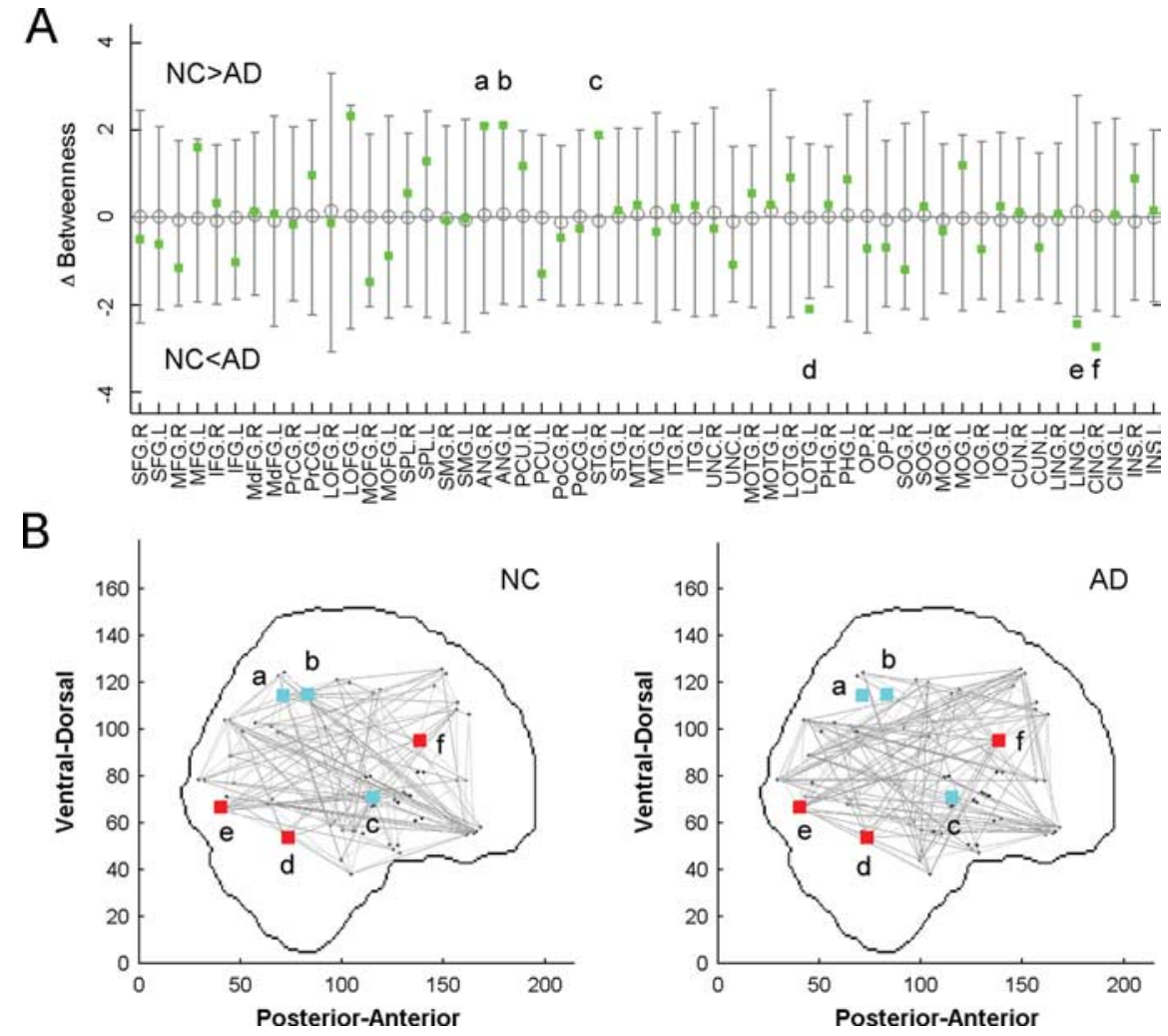

Figure 6. AD-related changes in nodal betweenness centrality. $\boldsymbol{A}$, The graph shows the differences (green square) in normalized betweenness $b_{i}$ for each region between the controls and AD patients. The gray circles and bar lines represent the mean values and 95\% confidence intervals of the between-group differences obtained from 1000 permutation tests, respectively. Significant decreases in $b_{i}$ in the AD patients were found in the right ANG $(a)$, left ANG $(b)$, and right STG $(c)$, and significant increases in the left LOTG $(d)$, left LING $(g)$, and right cingulate $(h)$. $\boldsymbol{B}$, Regions showing significant AD-related changes in $b_{i}$ were mapped to anatomical space in the controls (left) and AD (right) groups. Regions showing AD-related decreases are colored in cyan and regions showing AD-related increases are colored in red. The black lines represent the links of the networks. Note that these results were obtained from the brain networks with a sparsity of $13 \%$. NC, Normal controls. For the abbreviations of regions, see supplemental Table 1 (available at www.jneurosci.org as supplemental material). tially affected at the onset of AD before sensory or motor regions (for review, see Delbeuck et al., 2003). Gomez-Isla et al. (1997) reported $\mathrm{AD}$-related neuronal loss in the STG that paralleled the chronological evolution of dementia and correlated with the neurofibrillary tangles formation. Other researchers also observed $\mathrm{AD}$-related reduction of functional connectivity in the regions (Bokde et al., 2006; Wang et al., 2006). Thus, we speculated that these regional abnormalities may cause a segregation of different brain systems and yield a disruptive integration of large-scale brain networks. However, we also observed ADrelated increases in nodal centrality in several unimodal association cortex regions (e.g., LG and LOTG). Previous studies have suggested that these regions usually retain their functional capacity in the early stages of AD (Grady et al., 1988; Mentis et al., 1996). There is also additional evidence for AD-related increases in activation (Backman et al., 2000; Prvulovic et al., 2002; He et al., 2007a) and functional connectivity (Horwitz et al., 1995; Grady et al., 2003) in these regions, which has been characterized as compensatory recruitment of cognitive resources to maintain task performance. One could therefore speculate those increased regional centrality in $\mathrm{AD}$ might represent a compensatory process for the reduced centrality in other regions described previously. Overall, our results demonstrated AD-related changes in the nodal ability to manage information flow of cortical networks. cortical networks, suggesting a stronger local specialization. Given that the small-world model reflects an optimal balance between local specialization and global integration as described previously, the longer paths combined with higher clustering in the $\mathrm{AD}$ networks indicate a disturbance of the normal balance and make their networks more in favor of a regular configuration. It has been suggested that regular lattices have reduced signal propagation speed and synchronizability compared with small-world networks (Strogatz, 2001). Therefore, the ADrelated changes in small-world parameters reported here may reflect a less optimal topological organization, thus providing implications for the understanding of the relationship between network topology and neuropathological state of disease.

Altered nodal centrality in structural cortical networks in AD The AD networks were also found to show significantly decreased nodal centrality in several heteromodal association cortex regions (e.g., STG and ANG). Evidence from monkeys has demonstrated that STG and ANG have wide anatomical connections to the temporolimbic and neocortical association areas located in the prefrontal and parietal cortices (Seltzer and Pandya, 1994; Pandya, 1995) and the anterior temporal and prefrontal regions (Mesulam, 1998), respectively. Functional neuroimaging studies in humans have also suggested that these regions tend to have long-distance connections (Achard et al., 2006) and are preferen-

\section{Topological vulnerability in structural cortical networks in $\mathrm{AD}$}

Recent studies have demonstrated that small-world brain networks with embedded hubs exhibit surprising resilience to random failures and targeted attacks (Kaiser and Hilgetag, 2004; Achard et al., 2006). Assuming that dynamic behavior of a network is strongly associated with its fundamental topological organization, it seems reasonable to suppose that the alterations in network parameters would reflect the disruptions in the general performance of the network such as stability and robustness. This hypothesis was supported by our findings that the AD networks were extremely vulnerable to targeted attacks on its pivotal nodes and links compared with controls. The reduced topological stability might be attributed to pathological cortical organization in $\mathrm{AD}$ such as the aberrant cortical couplings, small-world architecture, and nodal centrality shown previously.

\section{Relationships between structural and functional brain networks}

A question of interest is whether the current findings of $\mathrm{AD}$ related alterations in structural cortical networks can be related to functional deficits in patients. In the mammalian neocortex, Honey et al. (2007) have found that the spontaneous neuronal dynamics can be structured at multiple temporal scales, suggesting a tight association between structural and functional net- 
works. In the human brain, we recently demonstrated that structural cortical networks (He et al., 2007b) share many common topological features (e.g., smallworld topology and hub nodes) with spontaneous low-frequency $(<0.08 \mathrm{~Hz})$ functional networks derived from fMRI data (Achard et al., 2006). One could therefore speculate that the AD-related alterations in structural networks shown here are likely to underlie functional impairments associated with this disease. Together, the present study strongly suggests that coordinated changes in structural features (cortical thickness) and their internal topologies are biologically meaningful, thus potentially opening up a new window into our understanding of cortical organization in $\mathrm{AD}$.

\section{References}

Achard S, Bullmore E (2007) Efficiency and cost of economical brain functional networks. PLoS Comput Biol 3:e17.

Achard S, Salvador R, Whitcher B, Suckling J, Bullmore E (2006) A resilient, low-frequency, small-world human brain functional network with highly connected association cortical hubs. J Neurosci 26:63-72.

Albert R, Jeong H, Barabasi AL (2000) Error and attack tolerance of complex networks. Nature 406:378-382.

Andrews TJ, Halpern SD, Purves D (1997) Correlated size variations in human visual cortex, lateral geniculate nucleus, and optic tract. J Neurosci 17:2859-2868.

Backman L, Almkvist O, Nyberg L, Andersson J (2000) Functional changes in brain activity during priming in Alzheimer's disease. J Cogn Neurosci 12:134-141.

Barrat A, Barthelemy M, Pastor-Satorras R, Vespignani A (2004) The architecture of complex weighted networks. Proc Natl Acad Sci USA 101:3747-3752.

Barton RA, Harvey PH (2000) Mosaic evolution of brain structure in mammals. Nature 405:1055-1058.

Bassett DS, Bullmore E (2006) Small-world brain networks. Neuroscientist 12:512-523.

Bassett DS, Meyer-Lindenberg A, Achard S, Duke T, Bullmore E (2006) Adaptive reconfiguration of fractal small-world human brain functional networks. Proc Natl Acad Sci USA 103:19518-19523.

Bohbot VD, Lerch J, Thorndycraft B, Iaria G, Zijdenbos AP (2007) Gray matter differences correlate with spontaneous strategies in a human virtual navigation task. J Neurosci 27:10078-10083.

Bokde AL, Lopez-Bayo P, Meindl T, Pechler S, Born C, Faltraco F, Teipel SJ, Moller HJ, Hampel H (2006) Functional connectivity of the fusiform gyrus during a face-matching task in subjects with mild cognitive impairment. Brain 129:1113-1124.

Buckner RL, Snyder AZ, Shannon BJ, LaRossa G, Sachs R, Fotenos AF, Sheline YI, Klunk WE, Mathis CA, Morris JC, Mintun MA (2005) Molecular, structural, and functional characterization of Alzheimer's disease: evidence for a relationship between default activity, amyloid, and memory. J Neurosci 25:7709-7717.

Bullmore ET, Woodruff PW, Wright IC, Rabe-Hesketh S, Howard RJ, Shuriquie N, Murray RM (1998) Does dysplasia cause anatomical dysconnectivity in schizophrenia? Schizophr Res 30:127-135.
Table 4. Regions showing AD-related changes in the betweenness centrality

\begin{tabular}{llll}
\hline & \multicolumn{2}{l}{ Normalized betweenness, $b_{i}$} & \\
\cline { 3 - 3 } Regions & $\mathrm{NC}$ & $\mathrm{AD}$ & \\
\hline Decreased nodal centrality in AD & & 0.06 \\
$\quad$ Right superior temporal gyrus & 1.96 & 0.01 & 0.04 \\
$\quad$ Left angular gyrus & 2.12 & 0.10 & 0.05 \\
$\quad$ Right angular gyrus & 2.19 & & 0.05 \\
Increased nodal centrality in AD & & 3.00 & 0.01 \\
$\quad$ Right cingulate gyrus & 0.03 & 2.33 & 0.04 \\
$\quad$ Left lateral occipitotemporal gyrus & 0.23 & 2.87 & 0.04 \\
$\quad$ Left lingual gyrus & 0.43 &
\end{tabular}

List of regions showed significant $(p<0.05)$ changes in the normalized nodal betweenness, $b_{j}$ in AD patients compared with controls. The regions were also highlighted in anatomical space in Figure 6. NC, Normal controls.
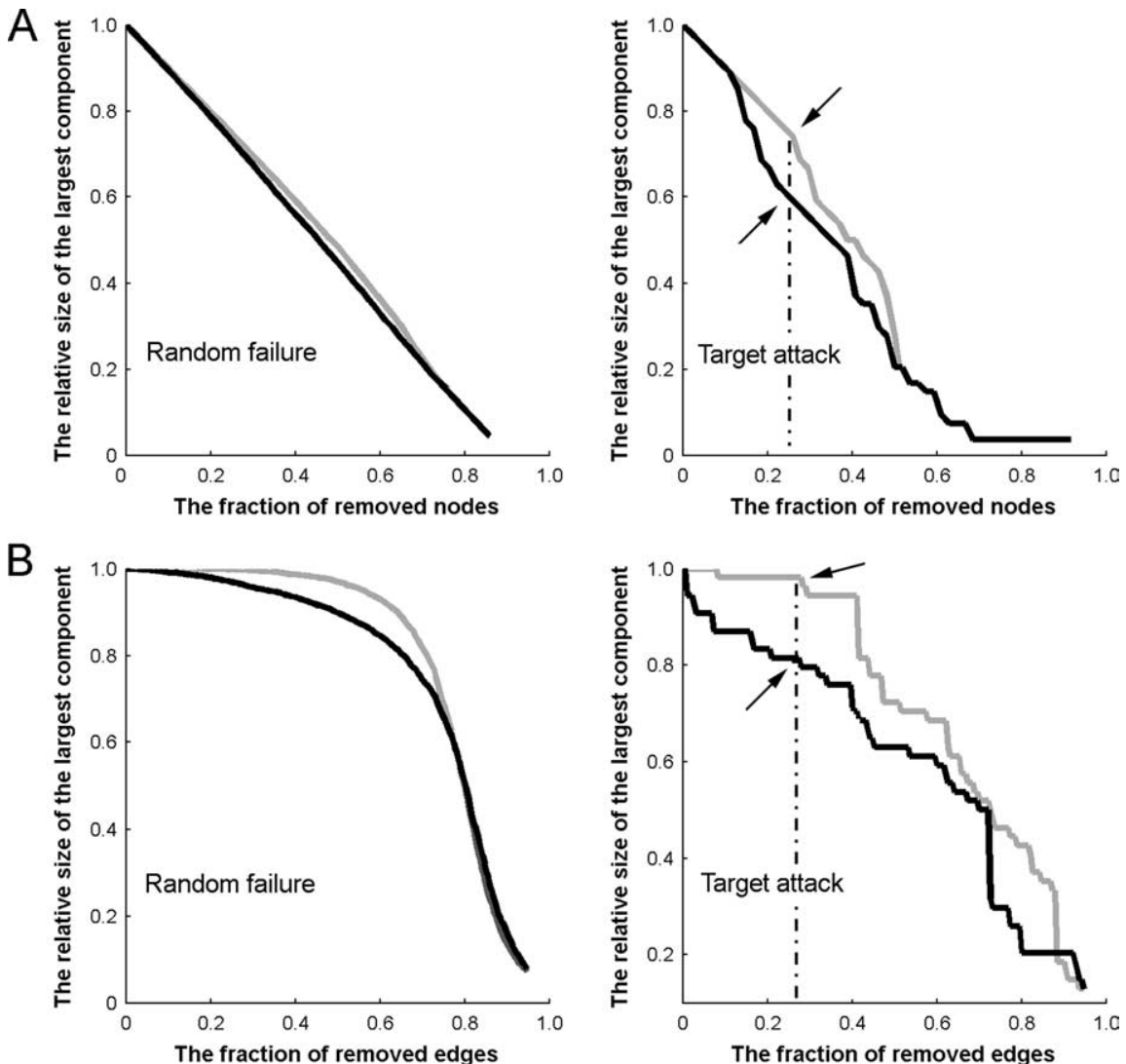

Figure 7. Topological robustness in structural cortical networks. The graphs show the relative size of the largest connected The brain network in the AD patients (black line) was approximately as robust as that in the controls (gray line) in response to random failures. However, it displayed remarkably reduced stability against targeted attack compared with the control network. Note that both brain networks used here had a same sparsity value of 13\%. NC, Normal controls.

Bullmore ET, Suckling J, Overmeyer S, Rabe-Hesketh S, Taylor E, Brammer MJ (1999) Global, voxel, and cluster tests, by theory and permutation, for a difference between two groups of structural MR images of the brain. IEEE Trans Med Imaging 18:32-42.

Celone KA, Calhoun VD, Dickerson BC, Atri A, Chua EF, Miller SL, DePeau K, Rentz DM, Selkoe DJ, Blacker D, Albert MS, Sperling RA (2006) Alterations in memory networks in mild cognitive impairment and Alzheimer's disease: an independent component analysis. J Neurosci 26:10222-10231.

Clark DA, Mitra PP, Wang SS (2001) Scalable architecture in mammalian brains. Nature 411:189-193.

Cohen J, Cohen P (1983) Applied multiple regression/correlation analysis for the behavioral sciences. Hillsdale, NJ: Erlbaum.

Collins DL, Neelin P, Peters TM, Evans AC (1994) Automatic 3D intersubject registration of MR volumetric data in standardized Talairach space. J Comput Assist Tomogr 18:192-205. 
Collins DL, Holmes CJ, Peters TM, Evans AC (1995) Automatic 3-D modelbased neuroanatomical segmentation. Hum Brain Mapp 3:190-208.

Delbeuck X, Van der Linden M, Collette F (2003) Alzheimer's disease as a disconnection syndrome? Neuropsychol Rev 13:79-92.

Ferrer I, Blanco R, Carulla M, Condom M, Alcantara S, Olive M, Planas A (1995) Transforming growth factor-alpha immunoreactivity in the developing and adult brain. Neuroscience 66:189-199.

Folstein MF, Folstein SE, McHugh PR (1975) “Mini-mental state.” A practical method for grading the cognitive state of patients for the clinician. J Psychiatr Res 12:189-198.

Fox MD, Snyder AZ, Vincent JL, Corbetta M, Van Essen DC, Raichle ME (2005) The human brain is intrinsically organized into dynamic, anticorrelated functional networks. Proc Natl Acad Sci USA 102:9673-9678.

Freeman LC (1977) A set of measures of centrality based upon betweenness. Sociometry 40:35-41.

Genovese CR, Lazar NA, Nichols T (2002) Thresholding of statistical maps in functional neuroimaging using the false discovery rate. NeuroImage 15:870-878.

Girvan M, Newman ME (2002) Community structure in social and biological networks. Proc Natl Acad Sci USA 99:7821-7826.

Gomez-Isla T, Hollister R, West H, Mui S, Growdon JH, Petersen RC, Parisi JE, Hyman BT (1997) Neuronal loss correlates with but exceeds neurofibrillary tangles in Alzheimer's disease. Ann Neurol 41:17-24.

Grady CL, Haxby JV, Schlageter NL, Berg G, Rapoport SI (1986) Stability of metabolic and neuropsychological asymmetries in dementia of the Alzheimer type. Neurology 36:1390-1392.

Grady CL, Haxby JV, Horwitz B, Sundaram M, Berg G, Schapiro M, Friedland RP, Rapoport SI (1988) Longitudinal study of the early neuropsychological and cerebral metabolic changes in dementia of the Alzheimer type. J Clin Exp Neuropsychol 10:576-596.

Grady CL, Furey ML, Pietrini P, Horwitz B, Rapoport SI (2001) Altered brain functional connectivity and impaired short-term memory in Alzheimer's disease. Brain 124:739-756.

Grady CL, McIntosh AR, Beig S, Keightley ML, Burian H, Black SE (2003) Evidence from functional neuroimaging of a compensatory prefrontal network in Alzheimer's disease. J Neurosci 23:986-993.

Greicius MD, Krasnow B, Reiss AL, Menon V (2003) Functional connectivity in the resting brain: a network analysis of the default mode hypothesis. Proc Natl Acad Sci USA 100:253-258.

Greicius MD, Srivastava G, Reiss AL, Menon V (2004) Default-mode network activity distinguishes Alzheimer's disease from healthy aging: evidence from functional MRI. Proc Natl Acad Sci USA 101:4637-4642.

Hagmann P, Kurant M, Gigandet X, Thiran P, Wedeen VJ, Meuli R, Thiran JP (2007) Mapping human whole-brain structural networks with diffusion MRI. PLoS ONE 2:e597.

Haxby JV, Grady CL, Koss E, Horwitz B, Heston L, Schapiro M, Friedland RP, Rapoport SI (1990) Longitudinal study of cerebral metabolic asymmetries and associated neuropsychological patterns in early dementia of the Alzheimer type. Arch Neurol 47:753-760.

He Y, Wang L, Zang Y, Tian L, Zhang X, Li K, Jiang T (2007a) Regional coherence changes in the early stages of Alzheimer's disease: a combined structural and resting-state functional MRI study. NeuroImage 35:488-500.

He Y, Chen ZJ, Evans AC (2007b) Small-world anatomical networks in the human brain revealed by cortical thickness from MRI. Cereb Cortex 17:2407-2419.

Hilgetag CC, Burns GA, O’Neill MA, Scannell JW, Young MP (2000) Anatomical connectivity defines the organization of clusters of cortical areas in the macaque monkey and the cat. Philos Trans R Soc Lond B Biol Sci 355:91-110.

Honey CJ, Kotter R, Breakspear M, Sporns O (2007) Network structure of cerebral cortex shapes functional connectivity on multiple time scales. Proc Natl Acad Sci USA 104:10240-10245.

Horwitz B, Grady CL, Schlageter NL, Duara R, Rapoport SI (1987) Intercorrelations of regional cerebral glucose metabolic rates in Alzheimer's disease. Brain Res 407:294-306.

Horwitz B, McIntosh AR, Haxby JV, Furey M, Salerno JA, Schapiro MB, Rapoport SI, Grady CL (1995) Network analysis of PET-mapped visual pathways in Alzheimer type dementia. NeuroReport 6:2287-2292.

Huang H, Zhang J, Jiang H, Wakana S, Poetscher L, Miller MI, van Zijl PC, Hillis AE, Wytik R, Mori S (2005) DTI tractography based parcellation of white matter: application to the mid-sagittal morphology of corpus callosum. NeuroImage 26:195-205.

Jiang T, He Y, Zang Y, Weng X (2004) Modulation of functional connectivity during the resting state and the motor task. Hum Brain Mapp 22:63-71.

Kabani N, Le Goualher G, MacDonald D, Evans AC (2001) Measurement of cortical thickness using an automated 3-D algorithm: a validation study. NeuroImage 13:375-380.

Kaiser M, Hilgetag CC (2004) Edge vulnerability in neural and metabolic networks. Biol Cybern 90:311-317.

Kaiser M, Hilgetag CC (2006) Nonoptimal component placement, but short processing paths, due to long-distance projections in neural systems. PLoS Comput Biol 2:e95.

Kim JS, Singh V, Lee JK, Lerch J, Ad-Dab'bagh Y, MacDonald D, Lee JM, Kim SI, Evans AC (2005) Automated 3-D extraction and evaluation of the inner and outer cortical surfaces using a Laplacian map and partial volume effect classification. NeuroImage 27:210-221.

Latora V, Marchiori M (2001) Efficient behavior of small-world networks. Phys Rev Lett 87:198701.

Lee JK, Lee JM, Kim JS, Kim IY, Evans AC, Kim SI (2006) A novel quantitative cross-validation of different cortical surface reconstruction algorithms using MRI phantom. NeuroImage 31:572-584.

Lerch JP, Evans AC (2005) Cortical thickness analysis examined through power analysis and a population simulation. NeuroImage 24:163-173.

Lerch JP, Pruessner JC, Zijdenbos A, Hampel H, Teipel SJ, Evans AC (2005) Focal decline of cortical thickness in Alzheimer's disease identified by computational neuroanatomy. Cereb Cortex 15:995-1001.

Lerch JP, Worsley K, Shaw WP, Greenstein DK, Lenroot RK, Giedd J, Evans AC (2006) Mapping anatomical correlations across cerebral cortex (MACACC) using cortical thickness from MRI. NeuroImage 31:993-1003.

MacDonald D, Kabani N, Avis D, Evans AC (2000) Automated 3-D extraction of inner and outer surfaces of cerebral cortex from MRI. NeuroImage 12:340-356.

Maguire EA, Gadian DG, Johnsrude IS, Good CD, Ashburner J, Frackowiak RS, Frith CD (2000) Navigation-related structural change in the hippocampi of taxi drivers. Proc Natl Acad Sci USA 97:4398-4403.

Marcus DS, Wang TH, Parker J, Csernansky JG, Morris JC, Buckner RL (2007) Open access series of imaging studies (OASIS): cross-sectional MRI data in young, middle aged, nondemented, and demented older adults. J Cogn Neurosci 19:1498-1507.

Maslov S, Sneppen K (2002) Specificity and stability in topology of protein networks. Science 296:910-913.

McAlonan GM, Cheung V, Cheung C, Suckling J, Lam GY, Tai KS, Yip L, Murphy DG, Chua SE (2005) Mapping the brain in autism. A voxelbased MRI study of volumetric differences and intercorrelations in autism. Brain 128:268-276.

Mechelli A, Friston KJ, Frackowiak RS, Price CJ (2005) Structural covariance in the human cortex. J Neurosci 25:8303-8310.

Medina D, DeToledo-Morrell L, Urresta F, Gabrieli JD, Moseley M, Fleischman D, Bennett DA, Leurgans S, Turner DA, Stebbins GT (2006) White matter changes in mild cognitive impairment and $\mathrm{AD}$ : a diffusion tensor imaging study. Neurobiol Aging 27:663-672.

Mentis MJ, Horwitz B, Grady CL, Alexander GE, VanMeter JW, Maisog JM, Pietrini P, Schapiro MB, Rapoport SI (1996) Visual cortical dysfunction in Alzheimer's disease evaluated with a temporally graded "stress test" during PET. Am J Psychiatry 153:32-40.

Mesulam MM (1998) From sensation to cognition. Brain 121:1013-1052.

Mitelman SA, Buchsbaum MS, Brickman AM, Shihabuddin L (2005) Cortical intercorrelations of frontal area volumes in schizophrenia. NeuroImage 27:753-770.

Morris JC (1993) The Clinical Dementia Rating (CDR): current version and scoring rules. Neurology 43:2412-2414.

Morris JC, Storandt M, Miller JP, McKeel DW, Price JL, Rubin EH, Berg L (2001) Mild cognitive impairment represents early-stage Alzheimer disease. Arch Neurol 58:397-405.

Newman MEJ (2003) The structure and function of complex networks. SIAM Rev Soc Ind Appl Math 45:167-256.

Pandya DN (1995) Anatomy of the auditory cortex. Rev Neurol (Paris) 151:486-494.

Pantel J, Schroder J, Jauss M, Essig M, Minakaran R, Schonknecht P, Schneider G, Schad LR, Knopp MV (1999) Topography of callosal atrophy 
reflects distribution of regional cerebral volume reduction in Alzheimer's disease. Psychiatry Res 90:181-192.

Parent A, Carpenter MB (1995) Human neuroanatomy. Baltimore: Williams and Wilkins.

Prvulovic D, Hubl D, Sack AT, Melillo L, Maurer K, Frolich L, Lanfermann H, Zanella FE, Goebel R, Linden DE, Dierks T (2002) Functional imaging of visuospatial processing in Alzheimer's disease. NeuroImage 17:1403-1414.

Pujol J, Soriano-Mas C, Alonso P, Cardoner N, Menchon JM, Deus J, Vallejo J (2004) Mapping structural brain alterations in obsessive-compulsive disorder. Arch Gen Psychiatry 61:720-730.

Raichle ME, MacLeod AM, Snyder AZ, Powers WJ, Gusnard DA, Shulman GL (2001) A default mode of brain function. Proc Natl Acad Sci USA 98:676-682.

Robbins S, Evans AC, Collins DL, Whitesides S (2004) Tuning and comparing spatial normalization methods. Med Image Anal 8:311-323.

Rose SE, Chen F, Chalk JB, Zelaya FO, Strugnell WE, Benson M, Semple J, Doddrell DM (2000) Loss of connectivity in Alzheimer's disease: an evaluation of white matter tract integrity with colour coded MR diffusion tensor imaging. J Neurol Neurosurg Psychiatry 69:528-530.

Salvador R, Suckling J, Coleman MR, Pickard JD, Menon D, Bullmore E (2005) Neurophysiological architecture of functional magnetic resonance images of human brain. Cereb Cortex 15:1332-1342.

Seltzer B, Pandya DN (1983) The distribution of posterior parietal fibers in the corpus callosum of the rhesus monkey. Exp Brain Res 49:147-150.

Seltzer B, Pandya DN (1994) Parietal, temporal, and occipital projections to cortex of the superior temporal sulcus in the rhesus monkey: a retrograde tracer study. J Comp Neurol 343:445-463.

Singh V, Chertkow H, Lerch JP, Evans AC, Dorr AE, Kabani NJ (2006) Spatial patterns of cortical thinning in mild cognitive impairment and Alzheimer's disease. Brain 129:2885-2893.

Sled JG, Zijdenbos AP, Evans AC (1998) A nonparametric method for automatic correction of intensity nonuniformity in MRI data. IEEE Trans Med Imaging 17:87-97.

Sporns O, Zwi JD (2004) The small world of the cerebral cortex. Neuroinformatics 2:145-162.

Sporns O, Tononi G, Edelman GM (2000) Theoretical neuroanatomy: re- lating anatomical and functional connectivity in graphs and cortical connection matrices. Cereb Cortex 10:127-141.

Sporns O, Chialvo DR, Kaiser M, Hilgetag CC (2004) Organization, development and function of complex brain networks. Trends Cogn Sci 8:418-425.

Stam CJ, Jones BF, Manshanden I, van Cappellen van Walsum AM, Montez T, Verbunt JP, de Munck JC, van Dijk BW, Berendse HW, Scheltens P (2006) Magnetoencephalographic evaluation of resting-state functional connectivity in Alzheimer's disease. NeuroImage 32:1335-1344.

Stam CJ, Jones BF, Nolte G, Breakspear M, Scheltens P (2007) Small-world networks and functional connectivity in Alzheimer's disease. Cereb Cortex 17:92-99.

Strogatz SH (2001) Exploring complex networks. Nature 410:268-276.

Sydykova D, Stahl R, Dietrich O, Ewers M, Reiser MF, Schoenberg SO, Moller HJ, Hampel H, Teipel SJ (2007) Fiber connections between the cerebral cortex and the corpus callosum in Alzheimer's disease: a diffusion tensor imaging and voxel-based morphometry study. Cereb Cortex 17:2276-2282.

Talairach T, Tournoux P (1988) Co-planar stereotaxic atlas of the human brain. New York: Thieme.

Vincent JL, Patel GH, Fox MD, Snyder AZ, Baker JT, Van Essen DC, Zempel JM, Snyder LH, Corbetta M, Raichle ME (2007) Intrinsic functional architecture in the anaesthetized monkey brain. Nature 447:83-86.

Wada Y, Nanbu Y, Koshino Y, Yamaguchi N, Hashimoto T (1998) Reduced interhemispheric EEG coherence in Alzheimer disease: analysis during rest and photic stimulation. Alzheimer Dis Assoc Disord 12:175-181.

Wang L, Zang Y, He Y, Liang M, Zhang X, Tian L, Wu T, Jiang T, Li K (2006) Changes in hippocampal connectivity in the early stages of Alzheimer's disease: evidence from resting state fMRI. NeuroImage 31:496-504.

Watts DJ, Strogatz SH (1998) Collective dynamics of "small-world" networks. Nature 393:440-442.

Wright IC, Sharma T, Ellison ZR, McGuire PK, Friston KJ, Brammer MJ, Murray RM, Bullmore ET (1999) Supra-regional brain systems and the neuropathology of schizophrenia. Cereb Cortex 9:366-378.

Zijdenbos AP, Forghani R, Evans AC (2002) Automatic "pipeline" analysis of 3-D MRI data for clinical trials: application to multiple sclerosis. IEEE Trans Med Imaging 21:1280-1291. 